\title{
The first example of a paraben-dependent antibody to an Rh protein
}

\author{
W. John Judd, Jill R. Storry, Thomas D. Annesley, Marion E. Reid, Michelle Bensette, \\ Sherry Waddington, LouAnn Dake, David Rohrkemper, and Ricardo Valdez
}

BACKGROUND: Parabens are added to a commercia LISS (C-LISS) to retard microbial growth. Parabendependent anti-Jk ${ }^{\mathrm{a}}$ has been detected by the use of C-LISS.

CASE REPORT: Serum from a D+ woman reacted in antiglobulin tests with RBCs stored (2-4 hours, $\left.22-25^{\circ} \mathrm{C}\right)$ in C-LISS (Löw and Messeter formulation, Immucor). Freshly prepared C-LISS-suspended RBCs did not react; nor did RBCs stored in LISS-additive reagents, PEG, saline, or homemade LISS.

RESULTS: Studies using C-LISS-stored RBCs revealed an antibody that reacted with $\mathrm{D}+$ and $\mathrm{rrV}+\mathrm{RBCs}$, but not with r'r, r' $r$, or rrV-VS-RBCs. All partial D RBC phenotypes tested reacted, as did $D+L W-, r^{G} r, r^{\prime \prime} G_{r}, r^{y} r$, $r^{\prime} s \mathrm{rV}+V S+$, and $r^{\prime}$ s $r V-V S+R B C s$. The active ingredient in C-LISS was propylparaben. Other LISS ingredients were not required; saline solutions of propylparaben, ethylparaben, methyl salicylate, 2-phenoxyethanol, and butylparaben were active. Methylparaben and methyl- $m$ hydroxybenzoate were inactive. Reactivity to C-LISSstored RBCs could not be inhibited by propylparaben. Reactivity with $\mathrm{D}+\mathrm{V}-$ and $\mathrm{D}-\mathrm{V}+\mathrm{VS}+\mathrm{RBC}$ was not separable by adsorption-elution.

CONCLUSIONS: This antibody likely detects a neoantigen formed between active compounds and RBC membranes. Review of the structure of active compounds suggests that proximity between methyl and hydroxyl groups is important for binding with RBC membranes. The role of $\mathrm{RhD}$ is unclear; no single portion of $\mathrm{RhD}$ protein appears to be implicated.
$\mathrm{E}$ sters of $p$-hydroxybenzoic acid (parabens) are added to a commercially available LISS(C-LISS) to retard microbial growth. We have described ex amples of anti-Jk ${ }^{\mathrm{a}}$ that react only when parabens or related compounds are present in the reaction mixture. ${ }^{1}$ Because active compounds had to be present in mixtures of serum plus RBCs for serologic reactivity to occur, Halima et al. ${ }^{2}$ suggested that these examples of anti-Jk $k^{\mathrm{a}}$ react by an immune complex mechanism. In this report, we describe the first example of a paraben-dependent antibody to an $\mathrm{Rh}$ protein that does not require the presence of paraben in the reaction milieu. Rather, this antibody appears to react with a neoantigen formed between propylparaben or related compounds and an Rh protein.

\section{CASE REPORT}

The patient is a 24-year-old untransfused woman who recently moved to the United States from Yemen. She was seen at the University of Michigan Health Systems (UMHS) for evaluation of primary infertility; she was treated with oral clomiphene citrate and Provera, and she subsequently conceived. This is the patient's first known pregnancy. We received blood samples for routine prenatal testing: $\mathrm{ABO}$ and $\mathrm{Rh}$ typing and tests for unexpected antibodies.

Her blood typed as group $A, R_{1} r(D+C+c+E-e+)$, and indirect antiglobulin tests (anti-IgG) for unexpected antibodies by using $R_{1} R_{1}$ and $R_{2} R_{2}$ RBCs stored in C-LISS were

ABBREVIATIONS: C-LISS = commercial LISS; UMHS = University of Michigan Health Systems.

From the Department of Pathology, University of Michigan, Ann Arbor, Michigan; and the New York Blood Center, New York, New York.

Address reprint requests to: W. John Judd, FIBMS, MIBiol, Department of Pathology, UH-2G332, University of Michigan Hospitals, 1500 East Medical Center Drive, Ann Arbor, MI 481090054; e-mail: johnjudd@umich.edu.

Received for publication June 19, 2000; revision received September 11, 2000, and accepted September 14, 2000.

TRANSFUSION 2001;41:371-4. 
positive. The serum did not react in initial antibody identification tests performed with RBCs freshly prepared in CLISS. However, an apparent anti-D+V/VS was detected by the IAT using RBCs stored for 2 to 4 hours in C-LISS. Moreover, while the DAT was negative, an autocontrol performed with the patient's RBCs that had been stored in C-LISS was positive (2+).

The outcome of this patient's pregnancy is unknown. During this investigation, she was lost to follow-up.

\section{MATERIALS AND METHODS}

The LISS solution containing methylparaben and propylparaben is available commercially (Immucor Inc., Norcross, GA). This reagent is based on the original formulation described by Löw and Messeter in $1976,{ }^{3}$ and it contains glycine, sodium chloride, and phosphate buffer to give a final pH of 6.7 and an osmolality of $289 \pm 10$ mOsm per kg. Methylparaben and propylparaben are added to this solution by the manufacturer at concentrations of 0.6 g per $L$ and 0.1 g per L, respectively. ${ }^{4}$ The LISS solution is used for the detection of unexpected antibodies in routine pretransfusion and perinatal testing. Briefly, RBCs are suspended in the LISS solution at a concentration of 2 percent. Equal volumes of RBCs and serum are incubated at $37^{\circ} \mathrm{C}$ for 10 minutes, after which the RBCs are washed and tested with anti-IgG. ${ }^{4}$

RBCs used in this investigation were obtained from commercial RBC panels, volunteer blood donors, or the frozen rare RBC panels of our institutions. Many of the latter were obtained through the Serum, Cells and Rare Fluids International Exchange Program.

LISS additive reagents, PEG, ficintreated RBCs, and an acid elution test kit were from Immucor. Antiglobulin reagents and anti-IgG gel cards were from Ortho Clinical Diagnostics (Raritan, NJ).

In inhibition tests, serial 1-in-2 dilutions of the patient's serum were mixed with an equal volume of 0.1 -percent propylparaben in saline. After 30-minute incubation at room temperature, each dilution was tested against $R_{2} R_{2}$ RBCs that had been incubated in C-LISS. All other serologic procedures were performed according to the reagent manufacturers' directions or as described elsewhere. ${ }^{4}$ Agglutination reactions were graded and scored as described by Marsh. ${ }^{5}$ Solutions of parabens and re- lated compounds were made in our in-house prepared LISS (Löw and Messeter formulation) or in pH 7.3 PBS as described previously. ${ }^{1}$

\section{RESULTS}

The initial resolution of the positive screening test for unexpected antibodies was hampered by the fact that RBCs stored in C-LISS reacted, but those freshly suspended in CLISS did not react. The RBCs used for antibody detection at UMHS are prepared as 2-mL volumes in C-LISS and used within 4 hours. In contrast, RBCs used for antibody identification are suspended in C-LISS and tested immediately. By use of a panel of RBCs previously incubated with C-LISS (2 hours at $22-25^{\circ} \mathrm{C}$ ), an antibody with $\mathrm{D}+\mathrm{V} / \mathrm{VS}$ specificity was identified. However, while an autocontrol (patient's RBCs incubated in C-LISS) reacted, the DAT did not.

Table 1 summarizes the results of tests with RBCs of common and uncommon Rh phenotypes incubated in CLISS. Our patient's antibody reacted with most partial D RBCs to the same degree as it did with RBCs carrying a nor-

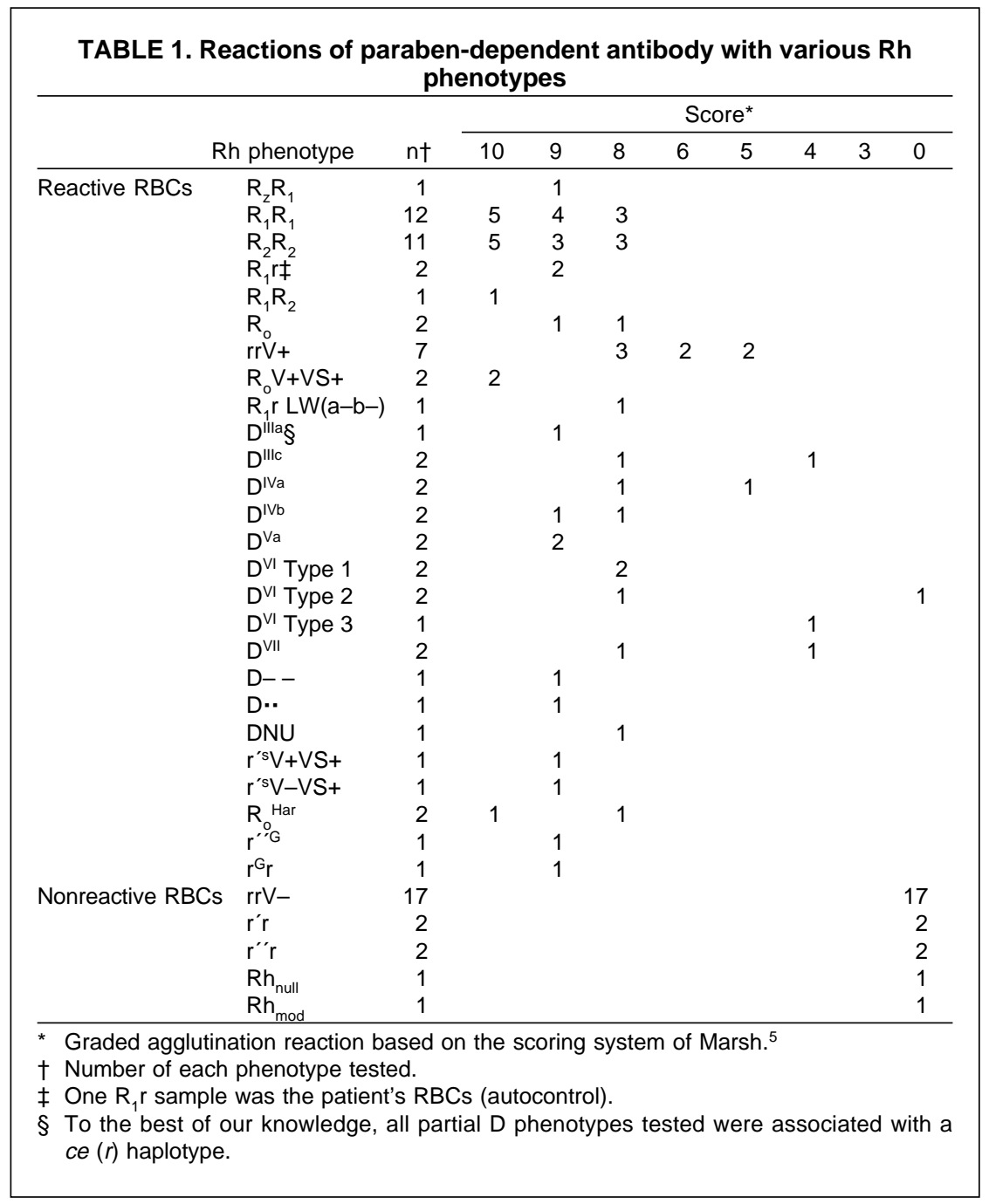


mal D, and it reacted with D- RBCs that are either G+, $\mathrm{V}+\mathrm{VS}+$, or V-VS+. Surprisingly, while $\mathrm{r}^{\prime} \mathrm{r}$ and $\mathrm{r}^{\prime \prime} \mathrm{r}$ RBCs did not react, $\mathrm{r}^{\mathrm{y}} \mathrm{r}$ RBCs reacted.

The active ingredient in C-LISS proved to be propylparaben. The other ingredients of C-LISS were not required, as antibody reactivity was not demonstrable by use of an inhouse preparation of Löw and Messeter's LISS ${ }^{3}$ to which no parabens were added, and it could be demonstrated with RBCs incubated in 0.1-percent propylparaben in normal saline. Reactivity with C-LISS-stored RBCs could not be inhibited by 0.1-percent (wt/vol) propylparaben. The activity against $\mathrm{D}+\mathrm{V}-$ and $\mathrm{D}-\mathrm{V}+\mathrm{VS}+\mathrm{RBC}$ coated with propylparaben was not separable by adsorption-elution. The anti-Rh activity was demonstrable in gel and ficin-antiglobulin tests, but only with RBCs previously incubated in C-LISS. Furthermore, and in contrast to the previously described examples of paraben anti-Jk ${ }^{\mathrm{a}}$, propylparaben did not have to be present in the reaction milieu for antibody detection; RBCs incubated in C-LISS and then washed and tested by gel reacted.

Methylparaben is also added to C-LISS. However, D+ RBCs incubated in methylparaben in either LISS or saline and then tested did not react. D+ RBCs incubated in a number of commercially available LISS additive solutions or PEG also did not react.

Figure 1 shows the structure of propylparaben and related compounds that were studied. Those compounds shown across the top of the figure were all capable of manifesting the paraben-dependent anti-Rh. Along the bottom are the structures of the $p, m$, and $o$ forms of methyl

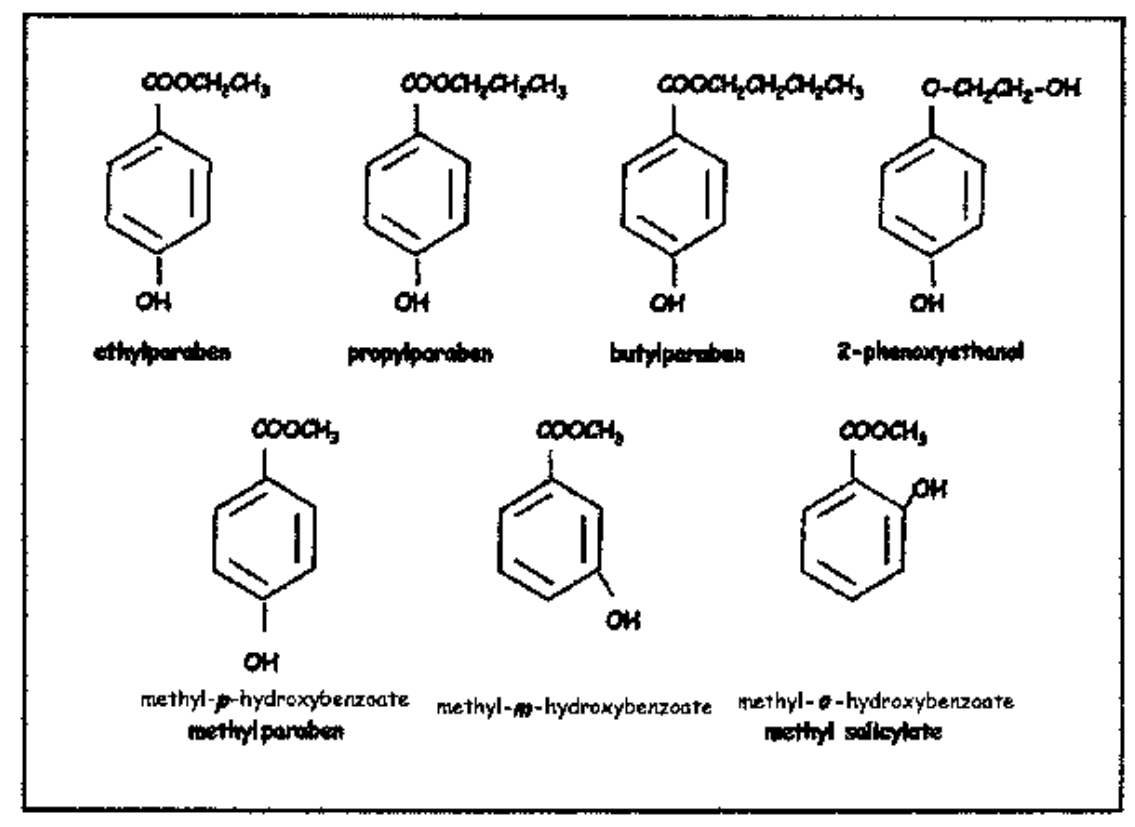

Fig. 1. Structures of parabens and related compounds used. All those on the top row reacted; on the bottom row, methylparaben and methyl- $m$-hydroxybenzoate did not react, and methyl salicylate reacted. hydroxybenzoate. Methyl- $p$-hydroxybenzoate (methylparaben) and methyl- $m$-hydroxybenzoate were inactive compounds, whereas methyl-o-hydroxybenzoate (methyl salicylate, Wintergreen) reacted.

\section{DISCUSSION}

Ohsako and Matsumoto ${ }^{6}$ showed that derivatives of hydroxybenzoates are transported across the RBC membrane and suggested that Band 3 (anion exchanger, AE1), a multipass membrane glycoprotein, ${ }^{7}$ is the transport protein. It is of interest that, on the basis of the presumed topology, Rh is also predicted to be a multipass transport protein that associates with Band 3 in the RBC membrane. ${ }^{8,9}$ It is of further interest, in light of previous reports of parabendependent anti-Jk ${ }^{\mathrm{a}},{ }^{1,2}$ that the urea transporter protein, which serves as the carrier molecule for the Kidd blood group antigens, is a multipass protein. ${ }^{10}$ It is tempting to speculate, therefore, that propylparaben and related reactive compounds bind to an Rh protein or to an epitope derived from the interaction of Rh with another RBC membrane protein, to form a neoantigen that is recognized by the antibody present in our patient's serum. This neoantigen hypothesis is somewhat analogous to the mechanism proposed for some cephalosporin-induced autoantibodies. $^{11}$

An initial review of the chemical structure of the active parabens (Fig. 1) suggested that ethylene $\left(\mathrm{CH}_{2}-\mathrm{CH}_{3}\right)$ groups contribute to this binding. However, $\mathrm{CH}_{2}-\mathrm{CH}_{3}$ groups are not present in the alkyl chains of methyl salicylate and 2phenoxyethanol, which also are active compounds.

It is plausible that the binding of phenol derivatives to RBC membranes is not directly related to a specific chemical group. In fact, our data suggest that the binding of phenol derivatives is related to their weak hydrophobicity (e.g., increased lipophilic characteristics), which would result in a stronger association with both the bilayer lipid membranes of RBCs and the multipass Rh protein that resides within the lipid membranes.

It is known that alkyl groups of phenol derivatives can shield the hydroxyl group and that this makes the derivative less hydrophilic, or more lipophilic (i.e., increases their binding to lipids). ${ }^{12}$ With methylparaben, which has a relatively short alkyl group, there is little shielding. As the length of the alkyl substitution on the carboxyl group of paraben increases beyond the methyl group (see Fig. 1), 
there can be better interaction with the $p$-hydroxyl group. In the case of methyl salicylate, in which the methyl and hydroxyl groups are in close proximity to each other, this shielding readily occurs. This hypothesis is supported by the fact that methyl salicylate (o-alkyl group) is one-fourth as soluble in water as is methylparaben ( $p$-alkyl group).$^{13} \mathrm{It}$ is interesting that clomiphene citrate, which our patient was receiving, has a purported metabolite containing a phenyl group with adjacent methoxy and hydroxyl groups in the same configuration as those of methyl salicylate. ${ }^{14,15}$

Unfortunately, the data on Rh variant RBCs shed little light on the precise role of the Rh proteins RhD and RhCE in the binding of the paraben-dependent antibody. These tests were performed to determine if there is a specific portion of Rh protein that must be present for binding to occur. Initially, tests with RBCs of common Rh phenotypes suggested a specificity of anti-D plus anti-V and/or anti-VS, as $\mathrm{D}+, \mathrm{D}-\mathrm{V}+$ and $\mathrm{D}-\mathrm{VS}+\mathrm{RBCs}$ reacted, but RBCs of other common D- phenotypes did not. Further testing with RBCs of uncommon Rh phenotypes showed that the antibody reacted with cells expressing hybrid $\mathrm{Rh}$ proteins with only small portions derived from RHD. Such RBCs (e.g., $\mathrm{r}^{\mathrm{G}}, \mathrm{r}^{\prime}{ }^{\prime} \mathrm{G}$, and $\mathrm{r}^{\prime} \mathrm{s}$ ) are not considered to be $\mathrm{D}+$. With partial $\mathrm{D}$ phenotype RBCs, the reaction strength was variable among RBCs from the same category, and it did not appear to be related to the number of $\mathrm{D}$ epitopes expressed (e.g., $\mathrm{R}_{\mathrm{o}}{ }^{\mathrm{Har}}$ and some $\mathrm{D}^{\mathrm{VI}} \mathrm{RBCs}$ gave the same reactions as normal $\mathrm{D}+$ and $\mathrm{D}^{\mathrm{III}}$ RBCs).

Molecular studies have shown that many Rh phenotypes are the result of gene rearrangements encoding hybrid RhD/RhCE proteins. ${ }^{9}$ Comparison of the probable molecular basis of the RBC phenotypes that react with our patient's serum does not reveal a common determinant. While most reactive RBCs express a portion of RhD encoded by exon 2 of $R H D$, that is the only portion expressed on $\mathrm{r}^{\mathrm{G}}$ RBCs. In contrast, $\mathrm{Ro}^{\mathrm{Har}} \mathrm{RBCs}$, which also react, express only a portion of RhD derived from exon 5 of $R H D$.

The most intriguing finding of all is the reactivity of $\mathrm{r}^{\mathrm{y}} \mathrm{r}$ (CE/ce) RBCs in the face of negative tests with r'r (Ce/ce) and $r^{\prime \prime} \mathrm{r}$ (cE/ce) RBCs. It is assumed that the $r^{y}$ haplotype involves normal RHCE-encoding sequences specific for $\mathrm{C}$ and $\mathrm{E}$ antigen expression. However, the reactivity of $\mathrm{r}^{\mathrm{y}} \mathrm{RBCs}$ may indicate that $\mathrm{r}^{\mathrm{y}}$ can be encoded by a hybrid gene composed of portions of $R H D$ and RHCE.

\section{REFERENCES}

1. Judd WJ, Steiner EA, Cochran RK. Paraben-associated autoanti-Jk ${ }^{\mathrm{a}}$ antibodies. Three examples detected using commercially prepared low-ionic strength saline containing parabens. Transfusion 1982;22:31-5.

2. Halima D, Garratty G, Bueno R. An apparent anti-Jk ${ }^{\mathrm{a}}$ reacting only in the presence of methyl esters of hydroxybenzoic acid. Transfusion 1982;22:521-4.

3. Löw B, Messeter L. Antiglobulin test in low-ionic strength salt solution for rapid antibody screening and cross-matching. Vox Sang 1974;26:53-61.

4. Judd WJ. Methods in immunohematology, 2nd ed. Durham, NC: Montgomery Scientific Publications, 1994.

5. Marsh WL. Scoring of hemagglutination reactions. Transfusion 1972;12:352-3.

6. Ohsako M, Matsumoto Y. [Transport of drugs through human erythrocyte membrane. Structure-activity relationship of benzoic acid and its derivatives between membrane transport and partition coefficient] (Engl abstract). Yakugaku Zasshi 1992;112:742-9.

7. Tanner MJ. The structure and function of band 3 (AE1): recent developments. Mol Membr Biol 1997;14:155-65.

8. Beckmann R, Smythe JS, Anstee DJ, Tanner MJ. Functional cell surface expression of Band 3, the human red blood cell anion exchange protein (AE1), in K562 erythroleukemia cells: Band 3 enhances the cell surface reactivity of Rh antigens. Blood 1998;92:4428-38.

9. Huang CH, Liu PZ, Cheng JG. Molecular biology and genetics of the Rh blood group system. Semin Haematol 2000;37:150-65.

10. Olives B, Mattei MG, Huet M, et al. Kidd blood group and urea transport function of human erythrocytes are carried by the same protein. J Biol Chem 1995;270:15607-10.

11. Arndt PA, Leger RM, Garratty G. Serology of antibodies to second- and third-generation cephalosporins associated with immune hemolytic anemia and/or positive direct antiglobulin tests. Transfusion 1999;39:1239-46.

12. Dearden JC, Tubby JH. Proceedings: Ortho-effects in structure-activity studies. Shielding of hydroxyl by alkyl groups. J Pharm Pharmacol 1974;26(Suppl):73p-4p.

13. Budavari S, ed. The Merck index, ed 12. Rahway, NJ: Merck, 1996.

14. Ruenitz PC, Arrendale RF, George GD, et al. Biotransformation of the antiestrogen clomiphene to chemically reactive metabolites in the immature female rat. Cancer Res 1987;47:4015-9.

15. Ruenitz PC, Arrendale RF, Schmidt WF, et al. Phenolic metabolites of clomiphene: [(E,Z)-2-[4-(1,2-diphenyl-2chlorovinyl)phenoxy] ethyl]diethylamine. Preparation, electrophilicity, and effects in MCF 7 breast cancer cells. J Med Chem 1989;32:192-7. 